\title{
Erratum to: Identification of rust fungi (Pucciniales) on species of Allium in Australia
}

\author{
Alistair R. McTaggart ${ }^{1,2}$ - Roger G. Shivas ${ }^{3}$. Chanintorn Doungsa-ard ${ }^{3}$. \\ Terri L. Weese ${ }^{1} \cdot$ Dean R. Beasley $^{3} \cdot$ Barbara H. Hall $^{4} \cdot$ Dean A. Metcalf $^{5}$. \\ Andrew D. W. Geering ${ }^{1}$
}

Published online: 22 October 2016

(C) Australasian Plant Pathology Society Inc. 2016

Erratum to: Australasian Plant Pathol

DOI:10.1007/s13313-016-0445-0

The original version of this article unfortunately contained a mistake. The presentation of Table 1 was incorrect. The corrected table is given below.

The online version of the original article can be found at http://dx.doi. org/10.1007/s13313-016-0445-0.

$\triangle$ Alistair R. McTaggart

alistair.mctaggart@gmail.com

1 Queensland Alliance for Agriculture and Food Innovation,

Ecosciences Precinct, The University of Queensland, GPO Box 267,

Brisbane, QLD 4001, Australia

2 Department of Microbiology and Plant Pathology, Tree Protection Co-operative Programme (TPCP), Forestry and Agricultural Biotechnology Institute (FABI), University of Pretoria, Private Bag X20, Pretoria 0028, South Africa

3 Plant Pathology Herbarium, Ecosciences Precinct, GPO Box 267, Brisbane QLD 4001, Australia

4 Plant Health and Biosecurity, SARDI, GPO Box 397, Adelaide, South Australia 5001, Australia

5 Metcalf Bio Control, 211Wyre Forest Road, Molesworth, TAS 7140, Australia 
Table 1 Basionyms for autoecious species of Puccinia and Uromyces described on species of Allium

\begin{tabular}{lllll}
\hline Name & Host & Year & Country & Reference \\
\hline Uredo porri & Allium porrum & 1809 & England & Sowerby (1809) \\
Xyloma allii & A. multiflorum & 1815 & France & de Candolle (1815) \\
Uredo alliorum & A. vineale, A. porri, A. multiflorum, Allium sp. & 1815 & France & de Candolle (1815) \\
Uredo ambigua & Allium sp. & 1815 & France & de Candolle (1815) \\
Puccinia allii & A. oleraceum, A. vineale & 1829 & Italy & Rudolphi (1829) \\
Puccinia alliorum & Nothoscordum gracile var. gracile (as A. fragrans) & 1840 & Portugal & Corda (1840) \\
Puccinia mixta & A. schoenoprasum & 1870 & Germany & Fuckel (1870) \\
Uromyces bicolor & A. validum & 1893 & USA & Coville (1893) \\
Puccinia blasdalei & A. serratum & 1893 & USA & Dietel (1893) \\
Uromyces aterrimus & A. validum, A. unifolii & 1895 & USA & Dietel (1895) \\
Puccinia granulispora & A. cernuum & 1895 & USA & Ellis and Everhart (1895) \\
Puccinia allii-japonici & A. japonicum & 1903 & Japan & Dietel (1903) \\
Uromyces durus & A. nipponicum & 1907 & Japan & Dietel (1907) \\
Puccinia allii-cepulae & A. cepa & 1946 & India & Mundkur and Thirumalachar (1946) \\
\hline
\end{tabular}

